\title{
Analgesic efficacy of zoledronic acid and its effect on functional status of prostate cancer patients with metastasis
}

\author{
Rafael Gálvez' \\ Victoria Ribera ${ }^{2}$ \\ José Ramón González-Escalada ${ }^{3}$ \\ Alicia Souto ${ }^{4}$ \\ María Luz Cánovas ${ }^{4}$ \\ Andrés Castro ${ }^{4}$ \\ Begoña Herrero ${ }^{5}$ \\ María de los Ángeles Maqueda ${ }^{5}$ \\ Matilde Castilforte ${ }^{6}$ \\ José Javier Marco-Martínez \\ Concepción Pérez ${ }^{8}$ \\ Lorenza Vicente-Fatela ${ }^{9}$ \\ Consuelo Nieto MD ${ }^{10}$ \\ Maria José Orduña" \\ Anna Padrol'2 \\ Enrique Reig ${ }^{13}$ \\ Joaquín Carballido'4 \\ José Manuel Cózar ${ }^{15}$ \\ 'Pain and Palliative Care Unit, Hospital \\ Universitario Virgen de las Nieves, Granada, \\ Spain; ${ }^{2}$ Pain Unit, Hospital Vall d'Hebron, \\ Barcelona, Spain; ${ }^{3}$ Pain Unit, Hospital \\ Ramón y Cajal, Madrid, Spain; ${ }^{4}$ Pain Unit, \\ Hospital Cristal Piñor, Ourense, Spain; \\ ${ }^{5}$ Pain Unit, Hospital Virgen de la Macarena, \\ Sevilla, Spain; ${ }^{6}$ Pain Unit, Fundación Jiménez \\ Díaz, Madrid, Spain; ${ }^{7}$ Pain Unit, Hospital \\ Príncipe de Asturias, Alcalá de Henares, \\ Madrid, Spain; ${ }^{8}$ Pain Unit, Hospital La \\ Princesa, Madrid, Spain; ' $P$ Pain Unit, Hospital \\ Universitario 12 de Octubre, Madrid, Spain; \\ ${ }^{10}$ Pain Unit, Fundación Alcorcón, Madrid, \\ Spain; "Pain Unit, Hospital Xeral-Calde, \\ Lugo, Spain; ${ }^{12}$ Pain Unit, Hospital Joan XXIII, \\ Tarragona, Spain; ${ }^{13}$ Pain Unit, Hospital Puerta \\ del Hierro, Madrid, Spain; ${ }^{14}$ Urology Service, \\ Hospital Puerta del Hierro, Madrid, Spain; \\ ${ }^{15}$ Urology Service, Hospital Universitario \\ Virgen de las Nieves, Granada, Spain
}

Correspondence: Rafael Gálvez Unidad del Dolor y Cuidados Paliativos, Hospital Universitari Virgen de las Nieves, 18012 Granada, Spain

Tel +3495802 0646;

Mobile +34619073839

Fax +349582908778

Email rafaelgalvez@hotmail.com
Objectives: A multi-centered observational study evaluated the efficacy of zoledronic acid for improving pain and mobility, and preventing skeletal-related events (SRE) (fracture, spinal compression, pain-relieving radiotherapy), in patients with prostate cancer and bone metastasis.

Materials and Methods: Males $(\mathrm{n}=218)$ with prostate cancer and bone metastasis undergoing oncologic therapy received zoledronic acid (4 mg iv/month) for 6 months. Parameters evaluated were: 1) pain and movement after 2 consecutive doses; 2) quality of life; 3) SRE incidence and time-to-appearance. Medication tolerance and treatment satisfaction were assessed using a questionnaire.

Results: A total of 170 that matched all the inclusion criteria (78\%) out of 218 were evaluable for efficacy. There was a measurable statistically significant reduction in pain at rest and on movement as well as an improvement in the quality of life compared with baseline. Best results were obtained with early treatment. Overall incidence of bone events was $11.2 \%$. Of the 212 patients (97.2\%) evaluable for safety, $16 \%$ suffered adverse events and $66 \%$ expressed satisfaction with the treatment

Discussion: Zoledronic acid is effective for reducing pain, improving mobility, and increasing the quality of life in patients with prostate cancer with bone metastasis. Its easy administration and good tolerability make zoledronic acid one of the principal therapeutic tools in the management of patients with pain associated with bone metastasis from prostate cancer.

Keywords: bone metastasis, pain, prostate cancer, zoledronic acid

\section{Introduction}

Bone metastasis from blood-borne dissemination and the introduction of tumor cells into the bone alters the balance between osteoblasts and osteoclast. Although most cases of bone metastasis are osteolytic metastasis with an increase in reabsorption, some cancers predominate in the osteoblasts, such as in the prostate, but in which osteolysis is also present (Clarke et al 1991; Garnero et al 2000; Berruti et al 2001; Scher 2003).

Between $30 \%$ and $50 \%$ of patients with prostate cancer have multiple bone metastasis because the diagnosis is made very late in the disease course (Berruti et al 2001). This pattern has not changed over the past 10 years. The incidence of bone metastasis is around $65 \%$ of patients with cancer of the prostate and also of those with breast cancer (Coleman 2001).

Although the clinical evolution of metastatic bone disease in prostate cancer is relatively protracted, the presence of metastasis is considered a sign of therapeutic failure, of hormone-resistance, and of tumor progression. The vital prognosis of the patients is obscured and morbidity - mortality is favored in the short to medium term (Berruti et al 2001; Coleman 2001; Scher 2003). Bone metastasis is one of the principal causes of pain in patients with advanced-stage prostate cancer as well as one of the main clinical features of incapacity. Further, it is one of the most important 
complications of the disease and the principal cause of the loss in quality of life (QoL).

The principal symptom of pain has very specific characteristics, ie, pain on movement which declines at rest but which, in the advanced stage of the disease, becomes constant. Pain is short and intense, at times superficially located, and is accompanied by contraction of the striated musculature proximal to the affected bone (Payne 1993). The increase in pain intensity in these patients when walking parallels the increase in the tumor progression. The onset of pain due to the metastasis is not easily foreseen and bears no direct relationship to the clinico-radiological status of the disease (Dreicer 1997).

Together with the pain due to bone metastasis, more severe pain is associated with skeletal complications, usually in subjects who are hormone resistant. Further, the patient's QoL is drastically altered when other aspects such as pathological fractures, hypercalcemia, spinal displacement and compression, and paraplegia are added (Scher 2003).

Therapeutic options are limited when patients are in an advanced stage of prostate cancer. The options include radiotherapy, systemic endocrine hormone therapy, and cytotoxic drugs, but the benefit obtained is slight (Oh 2000). As recommended by the WHO (Rhiner and Kedziera 1999; Bruera), there is a need for adjuvant palliative therapy to control the principal symptom of pain when the pain persists despite the anti-tumor therapy.

Analgesic treatment of bone pain of metastatic origin is complex and often fails. Locally administered radiotherapy and/or of radioisotopes and analgesics are the options, together with the use of bisphosphonates. Bisphosphonates are an alternative strategy in the treatment of symptoms such as skeletal complications, even if they do not directly influence tumor progression and survival (Coleman 2000; Clamp et al 2004).

Bisphosphonates appear to be efficacious in bone metastasis, not only in alleviating pain and controlling tumor hypercalcemia, but also in pre-empting or minimizing adverse skeletal events (Averbuch 1993; Papapoulos et al 2000). They act as strong inhibitors of bone matrix reabsorption and, as such, are genuine suppressors of osteolysis. Osteoclastic expansion is reduced (inhibitor of tumor growth factor) and differentiation of macrophage - monocyte precursors is impeded (Coleman 1991; Warrel 1992; Rogers et al 1997; Major et al 2000).

Zoledronic acid is a third-generation bisphosphonate, with a side-chain that contains an imidazole ring. It has 4 principal characteristics (Warrel 1992; Boisser et al 2000; Tassone et al 2000; Small et al 2003):
- a strong inhibitor of osteoclastic bone reabsorption with a potency greater than that of first- and second-generation bisphosphonates;

- can be administered via the parenteral route, similar to its predecessor pamidronate, but is easier to manage and requires no more than 15 minutes for administration;

- approved by the FDA for the treatment of bone metastasis and also for adverse events resulting from the metastasis;

- use for bone metastasis resulting from prostate cancer is among its principal indications.

In an in vivo model of hypercalcemia induced in rats, zoledronic acid appears 850 -fold more potent than palmidronate and 4-fold more than etidronate or clodronate (Warrel 1992). FDA approval is based on the results of 3 large-scale international clinical trials with $>3,000$ patients with cancer of the prostate, lung, and breast, and multiple myeloma. One of the best clinical trials (Saad et al 2002) studied 600 patients with bone metastasis resulting from advanced prostate cancer. Patients treated with zoledronic acid had a significant reduction in the number of skeletalrelated events (SRE) (pathological fractures: vertebral or nonvertebral) and an alleviation in bone pain. All these were achieved with a minimum of toxicity compared with placebo. The patients received doses of $4 \mathrm{mg}$ iv every 4 weeks. Saad et al (2004) evaluated the long-term role of zoledronic acid in the prevention of skeletal events in metastatic prostate cancer patients.

We report a multi-centered, prospective, observational open trial to evaluate the efficacy of zoledronic acid in patients diagnosed as having prostate cancer with bone metastasis who were hormone-sensitive (HS; D-2 on the Whitmore-Jewett scale) or hormone-resistant (HR) in progression stage of the disease.

\section{Material and methods Design and study population}

The observational, multi-centered, prospective, and descriptive study was conducted in a group of patients with prostate cancer and bone metastasis receiving treatment with zoledronic acid. The principal objectives were to evaluate the improvement in pain management and functional status of the patient. Secondary objectives were tolerability and ease of administration of zoledronic acid.

\section{Inclusion period}

Between July 1, 2002 and July 30, 2004, 218 patients with prostate cancer and bone metastasis were recruited 
by 27 specialists in urology service, 15 specialists in pain units, and 2 specialists in oncology service from a total of 33 hospitals throughout Spain.

\section{Inclusion criteria}

All patients needed to be male, over 18 years of age, diagnosed as having adenocarcinoma of the prostate with histology confirmation, and who had undergone androgen deprivation. All patients were diagnosed with bone metastasis at recruitment based on bone scan and none of them received any prior treatment with bisphophonates.

The overall general status considered acceptable was Eastern Cooperative Oncology Group (ECOG) $\leq 2$ and Karnofsky Index $(\mathrm{KI}) \geq 70$, and the presence of metastatic bone pain on movement measured on a visual analog scale (VAS) of $\geq 4$ was essential for trial entry. Patients were not to have had, in the month before study commencement, any history of pathological fracture, episodes of spinal compression, or radiotherapy to the bone.

All patients could be classified into 2 subgroups well characterized with the clinical and biochemical criteria available. Patients classified as hormone-sensitive (HS) ( $\mathrm{n}=76,36 \%)$ had a sensitivity response to hormonal treatment determined by prostate-specific antigen (PSA) values behavior and by clinical response. Patients classified as hormone-resistant $(\mathrm{HR})(\mathrm{n}=135,64 \%)$ showed therapeutic refractoriness to hormone manipulation determined by PSA values (increase $>50 \%$ in 3 consecutive determinations), by the increasing analgesic demand, and the need to incorporate other anticancer treatments.

\section{Exclusion criteria}

Patients with hypersensitivity to zoledronic acid, to bisphosphonates, or to any excipients of the zoledronic acid formulation; patients with noncompliance to medications and those considered potentially unreliable; patients with altered renal function with levels of creatinine $>265 \mu \mathrm{mol} / \mathrm{L}(3 \mathrm{mg} / \mathrm{dL})$ or clinically significant hypercalcemia $(>45 \mathrm{mmol} / \mathrm{L}$; $16 \mathrm{mg} / \mathrm{dL}$ ); patients with deterioration in cognitive capacity and having difficulty in understanding the methodological requirements of the study. The antineoplasic therapy on trial entry and during the trial was at the discretion of the attending physician.

\section{Ethics}

All patients provided written informed consent and the study was conducted in accordance with the principles of Helsinki and the requirements of the Spanish government.

\section{Data collection and description of measures: efficacy variables}

Patients were extensively evaluated at baseline and at 6 and 12 months of follow-up after treatment commencement with zoledronic acid.

A complete physical examination was performed and a detailed clinical history taken. A bone scan was performed routinely unless a previous scan had been performed within 3 months and documented results were available. Blood and urine samples were taken for biochemical and hematological investigations. Pain at rest and on movement was assessed on the VAS. At the same visit all medications and analgesics already in use were recorded, the functional status on the ECOG scale and the KI was assessed, and the Lattinen and SF-36 questionnaires were completed

\section{Pain measures}

The principal variables were variation in pain and patient mobility using the VAS score to evaluate pain intensity at rest and on movement.

We used also the Lattinen scale composed of 5 categories: pain intensity, patient activity, pain frequency, use of analgesics, and hours of sleep. Each category comprises 5 items scored from 0 (better) to 5 (worse). Maximum score of the Lattinen scale is 20; a decrease in score indicates an improvement in patient status. Pain alleviation scores on the scale of 0 to 4 were: 0 (no alleviation), 1 (slight alleviation), 2 (moderate alleviation), 3 (considerable alleviation), 4 (complete alleviation).

\section{Quality of life measures}

Parameters of functional status and quality of life (QoL) are included in the Eastern Cooperative Oncology Group (ECOG) scale, the Karnofsky Index (KI), and the SF-36 questionnaire. Further, we used the questionnaire to assess patient satisfaction and reduction in analgesics use. The ECOG and KI status was evaluated every trimester. An increment in the ECOG and a decrease in the KI indicate a worsening of the patient's functional status. The QoL SF-36 questionnaire was completed by the patient every 3 months after inclusion into the study to evaluate their physical and mental status. The responses to each question were coded and recoded according to the "Manual of Scoring of the Spanish version of the Health Questionnaire SF-36" published by the Municipal Institute of Medical Investigation (MIMI) (Instituto Municipal de Investigación Médica [IMIM]). The overall results of the two scores derived from the questionnaire were translated to a scale from 0 (poorer 
health) to 100 (better health). An increase in score indicated an improvement in the patient's status.

The questionnaire on patient satisfaction (comprising 4 items) was completed during the last consultation visit of the study, or at the time the patient decided to abandon the study for whatever motive. At the clinical visits in the study, all adverse effects reported by the patient reported during the consultation were recorded, whether or not related to the study medication.

\section{Treatment employed}

Zoledronic acid was administered at a dose of $4 \mathrm{mg} /$ day iv infused over 15 minutes, every 4 weeks ( \pm 1 week). Treated patients were followed up to the end of the study. Calcium and vitamin D supplements (daily tablet equivalent to 500 $\mathrm{mg} /$ day calcium and $400 \mathrm{mg} /$ day of vitamin D) were provided during the bisphosphonate treatment. Patients needed to start taking these supplements before the start of the bisphosphonates treatment, and needed to receive at least 1 dose before the second consultation visit. The patients who abandoned the study before completing the treatment and for whom there had been at least 1 evaluation of status after the start of the study were considered evaluable on the intentionto-treat analysis, taking as reference the last evaluation of response available prior to the patient abandoning the study. All patients who received at least 1 dose of the study drug were included in the safety analysis.

\section{Duration of treatment and clinical consultations}

Duration of evaluated treatment was 6 dose administrations in an interval of 3-5 weeks and a total of 18-30 weeks. However, patients continued treatment with zoledronic acid after the 6 sessions if the attending physician considered it necessary. The first posttreatment assessment needed to be performed at 3 days after the first dose. This was done by telephone to check for possible adverse effects. Subsequent visits were scheduled for the first, second, third, fourth, fifth, and sixth month. An investigator deciding that changing the treatment away from the study drug would be in the patient's interest to was considered a valid treatment failure.

\section{Data analyses and statistical tests}

The variables are described using frequency tables and percentages for the qualitative variables, and included the mean, median, standard deviation, and range for the continuous variables. Comparisons of the variables of interest over the long term of the follow-up visits were carried out using the
Wilcoxon nonparametric test (comparison of baseline data with the rest of the follow-up visits using paired sample test). Comparisons between subgroups of the study sample were carried out using the Whitney-Mann nonparametric test ( 2 groups) or the Kruskal-Wallis test (more than 2 groups). Qualitative variables were analyzed using contingency tables $\left(\chi^{2}\right.$ test). All the statistical analyses were performed using the two-tailed test with $\alpha$ significance of 5\%. Efficacy was evaluated using the at-rest and on-movement VAS. The Lattinen scale values were analyzed over the long term of the follow-up visits using the Wilcoxon nonparametric test and the comparison between subgroups using the nonparametric Mann-Whitney U test. All statistical analyses were performed using the SPSS package (version 12.0).

\section{Results}

\section{Study sample description}

A total of 218 male patients were included in the study (mean age 71.5 years; SD 7.5) (Table 1). Principal sociodemographic and clinical characteristics of the population used in the evaluation of safety are those of the 212 patients who had received at least a single dose of zoledronic acid (Table 2). Of the cases, 170 (78.0\%) were considered evaluable for analysis of effectiveness; 48 cases were excluded for not fulfilling inclusion/exclusion criteria (Table 2).

Data on timing of prostate cancer and bone metastasis diagnosis in both subgroups of patients (HS and HR) are shown on Table 3.

\section{Pain}

Patients had moderate decrease in pain over the first month of treatment. As assessed on the VAS the at-rest scores decreased from 3.7 (SD 2.5) to 2.7 (SD 2.3) and reached 2.3 points (SD 2.3) at the third evaluation. The at-rest pain was maintained close to this score over the subsequent months. Differences between baseline score and the remaining months of follow-up were statistically significant (Wilcoxon test; $p<0.0001$ ) (Table 4). This effect was observed in $\mathrm{HS}$ and HR patients.

The on-movement VAS decreased from 6.0 (SD 2.0) to 4.6 (SD 2.7) at the end of the first month of treatment and to 4.2 (SD 2.7) over the second month, and remained constant until the end of the study. Differences between baseline and the rest of the months of follow-up were statistically significant (Wilcoxon test; $\mathrm{p}<0.0001$ ) (Table 5). No differences were observed between HS and HR for on-movement VAS decrease.

In the first month of treatment, $45.5 \%$ of the patients referred to the alleviation of pain as "none" or "slight" and 
Table I Baseline clinical and demographic data

\begin{tabular}{|c|c|c|}
\hline Characteristics & $\mathbf{N}$ & Percentage \\
\hline \multicolumn{3}{|l|}{ Age, years } \\
\hline Mean \pm SD & $71.5 \pm 7.5$ & \\
\hline Median & 73 & \\
\hline ECOG & $N=207$ & \\
\hline 0 & 16 & 7.7 \\
\hline I & 114 & 55.1 \\
\hline 2 & 63 & 6.8 \\
\hline$>2$ & 14 & 6.8 \\
\hline $\mathrm{KI}$ & $N=208$ & \\
\hline $100 \%-90 \%$ & 48 & 23.1 \\
\hline $80 \%$ & 84 & 40.4 \\
\hline $70 \%$ & 52 & 25.0 \\
\hline$<70 \%$ & 24 & 11.5 \\
\hline $\begin{array}{l}\text { Time since diagnosis } \\
\text { of bone metastases }\end{array}$ & $N=172$ & \\
\hline Mean years \pm SD & $0.9 \pm 1.2$ & \\
\hline Median & 0.5 & \\
\hline $\begin{array}{l}\text { Location of bone } \\
\text { metastases }\end{array}$ & $N=207$ & \\
\hline Vertebral column & 125 & 60.4 \\
\hline Ribs & 96 & 46.6 \\
\hline Pelvis & 86 & 41.5 \\
\hline Sacrum & 57 & 27.5 \\
\hline Femur & 53 & 25.6 \\
\hline
\end{tabular}

Abbreviation: KI, Karnofsky Index.

27.9 indicated "considerable" or "complete" alleviation. At study end (6 months), percentage of patients with "none" or "slight" alleviation declined to $33.8 \%$ while patients with "considerable" or "complete" alleviation increased to $55.1 \%$. Differences between baseline and follow-up were statistically significant (Wilcoxon test; $\mathrm{p}<0.05$ ).
The Lattinen index decreased from 9.0 points (SD 2.9) to 7.8 points (SD 3.6) at 2 months with a decrease during the first month of treatment to 7.8 points (SD 3.6), and remained constant over the remaining months of follow-up and reached a score of 7.6 points (SD 4.3) at the sixth month. Statistically significant differences were found for all months of follow-up (Mann-Whitney U test; $p<0.05$ ) as well as between baseline score and scores at the other months of follow-up visits in the overall study sample (Wilcoxon test; $\mathrm{p}<0.05)$. Patients were able to sleep an hour longer in the 6 months of treatment, starting with a mean of 6.7 hours/day (SD 2.1) and at ending with a mean of 7.5 hours (SD 2.0). These differences were statistically significant.

At the beginning of the study a total of 203 (95.8\%) patients received analgesic treatment, which declined by $10 \%$ after 4 months of treatment (Table 6 ).

At the start of the study, patients had a mean score on ECOG of 1.24 points (SD 0.57), at the third month 1.20 points (SD 0.75), and at the end of the follow-up period 1.31 points (SD 0.85). The differences for functional status did not reach statistical significance between the baseline visit and that at the third and sixth months. Also we did not observe any differences in these values between HS and HR (Table 7).

During the 6 months of follow-up, mean score score on KI was approximately 80 points, which indicates that patients had, over the whole study, a functional status of "good normal activity with some signs and symptoms of disease". Scores at baseline and those at the third and sixth months of follow-up did not differ significantly (Table 8).

The SF-36 questionnaire indicated a slight improvement in physical status. Significant differences were observed between baseline scores and those at the third and sixth months of follow-up. No changes were observed for

Table 2 Exclusion/inclusion criteria

\begin{tabular}{lll}
\hline & $\mathbf{n}$ & $\%$ \\
\hline Total recruited cases & 218 & $100.0 \%$ \\
Evaluable for efficacy & 170 & $78.0 \%$ \\
Not evaluable for efficacy & 48 & $22.0 \%$ \\
Without bone metastasis evidence according to bone scan & 4 & $1.8 \%$ \\
Nonacceptable general health status to accomplish follow-up visits (ECOG $>2$ or I Karnofsky <70\%) & 28 & $12.8 \%$ \\
Bone or metastatic pain absence (VAS on movement $<2$ ) & 10 & $2.6 \%$ \\
With pathological fracture or radiotherapy history one month prior to study & 2 & $0.9 \%$ \\
Altered renal function (creatinine $>265 \mu$ mol/L or $>3$ mg/dL) & 8 & $3.9 \%$ \\
Patient without any zoledronic acid dose & $9.1 \%$ \\
Patient without basal pain and mobility improvement and without skeletal events onset time & 9 \\
\hline
\end{tabular}

Abbreviation: VAS, visual analog scale. 
Table 3 Timing of prostate cancer diagnosis and bone metastasis

\begin{tabular}{|c|c|c|c|c|c|c|}
\hline & & $\mathbf{n}$ & Average & SD & Median & $\mathbf{p}$ \\
\hline \multirow[t]{3}{*}{ Time elapsed since bone metastasis diagnosis (years) } & HS & 68 & 0.6 & 0.8 & 0.3 & 0.0045 \\
\hline & $\mathrm{HR}$ & 103 & 1.1 & 1.4 & 0.7 & \\
\hline & Global & 172 & 0.9 & 1.2 & 0.5 & - \\
\hline \multirow[t]{3}{*}{ Time elapsed since prostate cancer diagnosis (years) } & HS & 57 & 1.1 & 2.1 & 0.5 & 0.0000 \\
\hline & $\mathrm{HR}$ & 92 & 3.3 & 2.8 & 2.3 & \\
\hline & Global & 150 & 2.5 & 2.8 & 1.4 & - \\
\hline
\end{tabular}

Abbreviations: HR, hormone resistant; HS, hormone sensitive; SD, standard deviation.

mental health between the baseline and the other followup visits.

At the end of the study at 6 months, $66.3 \%$ of the patients said they were "very or sufficiently satisfied" with the treatment administered, $22.4 \%$ were "moderately satisfied", and $11.2 \%$ "not satisfied", with a mean of $55.4 \%$, indicating that they had no objections to continuing with the treatment.

Table 4 Score evolution (at rest VAS)

\begin{tabular}{|c|c|c|c|c|}
\hline Global & $\mathbf{n}$ & SD & Median & $\mathbf{p}^{\mathrm{a}}$ \\
\hline Basal & 168 & 2.5 & 3.6 & - \\
\hline Month I & 169 & 2.3 & 2.0 & $<0.000$ I \\
\hline Month 2 & 169 & 2.4 & 2.0 & $<0.0001$ \\
\hline Month 3 & 169 & 2.3 & 2.0 & $<0.0001$ \\
\hline Month 4 & 169 & 2.4 & 2.0 & $<0.0001$ \\
\hline Month 5 & 169 & 2.6 & 2.0 & $<0.000$ I \\
\hline Month 6 & 169 & 2.6 & 2.0 & $<0.000$ I \\
\hline \multicolumn{5}{|c|}{ Hormone sensitive (HS) } \\
\hline Basal & 58 & 2.5 & 3.0 & - \\
\hline Month I & 58 & 2.2 & 1.9 & $<0.000$ I \\
\hline Month 2 & 58 & 2.2 & 1.7 & 0.0001 \\
\hline Month 3 & 58 & 2.1 & 1.3 & $<0.0001$ \\
\hline Month 4 & 58 & 2.1 & I.I & $<0.0001$ \\
\hline Month 5 & 58 & 2.3 & 0.8 & 0.0015 \\
\hline Month 6 & 58 & 2.4 & 0.7 & 0.0001 \\
\hline \multicolumn{5}{|c|}{ Hormone resistant (HR) } \\
\hline Basal & 110 & 2.4 & 4.0 & - \\
\hline Month I & 111 & 2.3 & 2.3 & 0.0008 \\
\hline Month 2 & 111 & 2.4 & 2.0 & $<0.000$ I \\
\hline Month 3 & 111 & 2.4 & 2.0 & $<0.0001$ \\
\hline Month 4 & 111 & 2.5 & 2.1 & 0.0053 \\
\hline Month 5 & 111 & 2.6 & 2.1 & 0.0034 \\
\hline Month 6 & III & 2.6 & 2.1 & 0.0038 \\
\hline
\end{tabular}

${ }^{a}$ Wilcoxon Test: Comparison for basal resting VAS.

Abbreviations: SD, standard deviation; VAS, visual analog scale.

\section{Adverse reaction}

The most frequent adverse reactions were arthralgias, shivering, fever, edema, and constipation. Only 2 patients had severe adverse reaction, both after the first dose: one had severe arthralgia and the other, who had a history of chronic renal insufficiency, suffered hypocalcemia, hyperphostatemia, and acute renal failure. The rest of the patients showed good tolerance to the drug (Table 9). Pain was associated

Table 5 Score evolution (on-movement VAS)

\begin{tabular}{|c|c|c|c|c|}
\hline Global & $\mathbf{n}$ & SD & Median & $\mathbf{p}^{\mathbf{a}}$ \\
\hline Basal & 169 & 2.0 & 6.0 & - \\
\hline Month I & 169 & 2.6 & 4.3 & $<0.0001$ \\
\hline Month 2 & 169 & 2.7 & 4.0 & $<0.000$ I \\
\hline Month 3 & 169 & 2.8 & 4.0 & $<0.000$ I \\
\hline Month 4 & 169 & 2.9 & 4.0 & $<0.0001$ \\
\hline Month 5 & 169 & 2.8 & 4.0 & $<0.000$ I \\
\hline Month 6 & 169 & 2.9 & 4.0 & $<0.0001$ \\
\hline \multicolumn{5}{|c|}{ Hormone sensitive (HS) } \\
\hline Basal & 58 & 1.9 & 5.0 & - \\
\hline Month I & 58 & 2.5 & 4.0 & $<0.000$ I \\
\hline Month 2 & 58 & 2.5 & 3.0 & $<0.000$ I \\
\hline Month 3 & 58 & 2.6 & 3.2 & 0.0001 \\
\hline Month 4 & 58 & 2.6 & 2.5 & $<0.000$ I \\
\hline Month 5 & 58 & 2.7 & 2.5 & $<0.000$ I \\
\hline Month 6 & 58 & 2.8 & 2.8 & 0.0009 \\
\hline \multicolumn{5}{|c|}{ Hormone resistant (HR) } \\
\hline Basal & 111 & 2.0 & 6.8 & - \\
\hline Month I & 111 & 2.6 & 5.0 & $<0.000$ I \\
\hline Month 2 & 111 & 2.7 & 4.0 & $<0.000 \mathrm{I}$ \\
\hline Month 3 & 111 & 2.8 & 4.3 & $<0.0001$ \\
\hline Month 4 & 111 & 2.9 & 5.0 & $<0.0001$ \\
\hline Month 5 & 111 & 2.8 & 4.3 & $<0.000$ I \\
\hline Month 6 & III & 2.9 & 4.9 & $<0.000 \mathrm{I}$ \\
\hline
\end{tabular}

aWilcoxon Test: Comparison for basal active VAS.

Abbreviations: SD, standard deviation; VAS, visual analog scale. 
Table 6 Pain treatment during follow-up

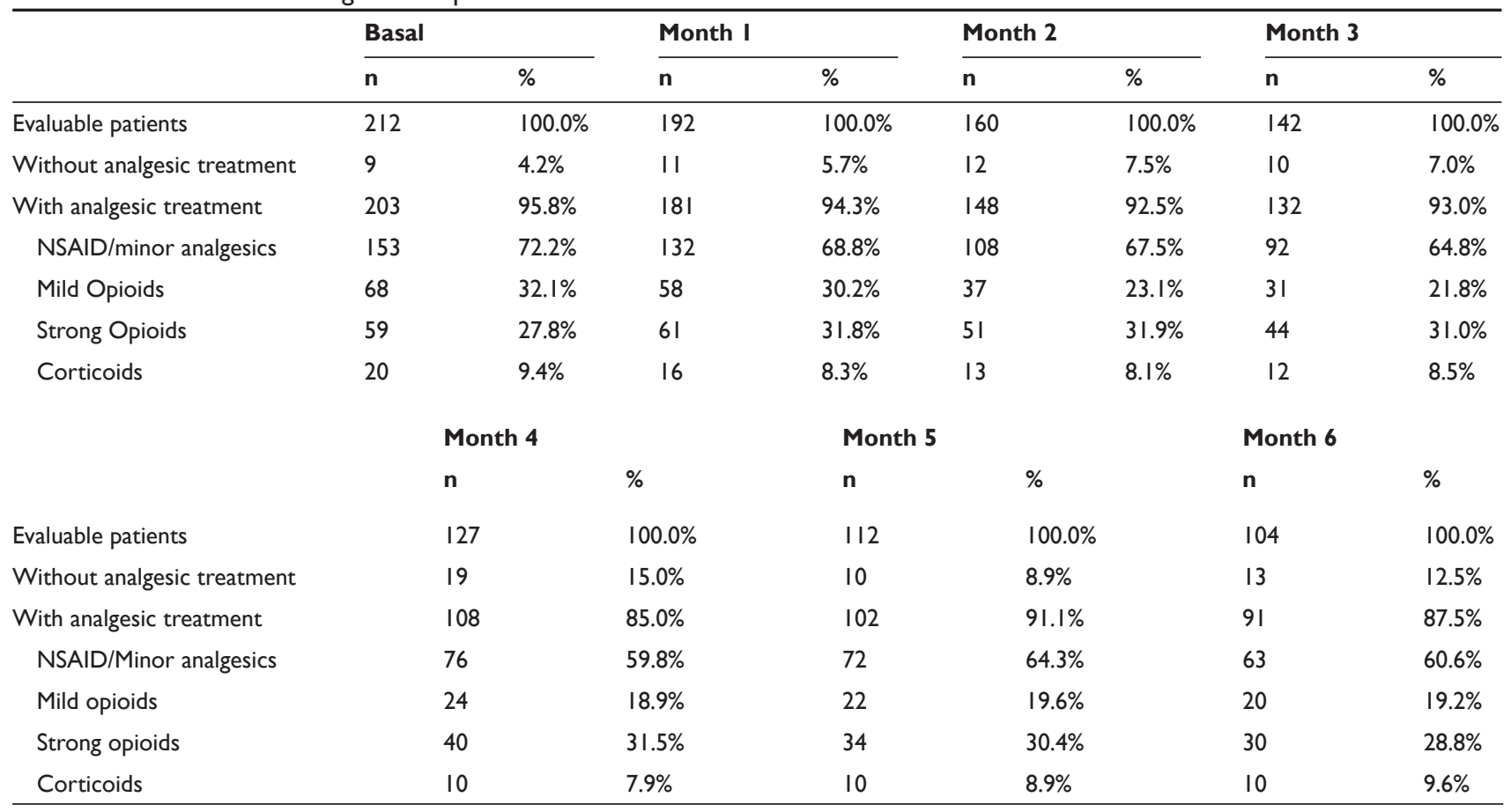

with bone metastasis and SRE. All of these were recorded in both HS and HR groups (Figure 1).

\section{Discussion}

Bone metastasis is the principal cause of morbidity in all types of cancer and the most-frequent cause of pain, especially that associated with movement. This pain results from release of prostaglandins causing a reduction in pain threshold, an increase in intra-bone pressure due to tumor invasion, and high periostial sensitivity. Pain from metastasis is often accompanied by limitation in the patient's functional status resulting from fractures and skeletal lesions, which cause a drastic loss in QoL (Levy 1993; Paye 1993; Cózar Olmo et al 1999).

The ideal management of pain of metastatic bone origin would be elimination of the tumor. However, this is not an option in these patients and, hence, the objective is pain reduction. The priority is to alleviate on-movement pain such as that resulting from skeletal-related events. However, the complex treatment and frequent failure indicates that the approach needs to be multi-disciplinary, using the whole arsenal available for this type of pain. In this approach,

Table 7 Functional status evolution according to ECOG scale

\begin{tabular}{|c|c|c|c|c|c|}
\hline & & $\mathbf{N}^{\mathbf{a}}$ & Average & SD & $\mathbf{p}^{\mathbf{b}}$ \\
\hline \multirow[t]{3}{*}{ Global } & Basal & 169 & 1.24 & 0.57 & - \\
\hline & Month 3 & 119 & 1.20 & 0.75 & 0.5687 \\
\hline & Month 6 & 81 & 1.31 & 0.85 & 0.1196 \\
\hline \multirow[t]{3}{*}{ Hormone sensitive (HS) } & Basal & 58 & 1.09 & 0.54 & - \\
\hline & Month 3 & 46 & 1.04 & 0.67 & 1.0000 \\
\hline & Month 6 & 35 & 1.11 & 0.76 & 0.4669 \\
\hline \multirow[t]{3}{*}{ Hormone resistant (HR) } & Basal & 111 & 1.32 & 0.57 & - \\
\hline & Month 3 & 73 & 1.30 & 0.79 & 0.5013 \\
\hline & Month 6 & 46 & 1.46 & 0.89 & 0.1664 \\
\hline
\end{tabular}

aln some cases no data were available.

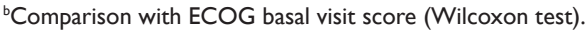

Abbreviation: SD, standard deviation. 
Table 8 Functional status evolution according to Karnofsky scale

\begin{tabular}{|c|c|c|c|c|c|}
\hline & & $\mathbf{n}^{\mathbf{a}}$ & Average & SD & $\mathbf{p}^{\mathrm{b}}$ \\
\hline \multirow[t]{3}{*}{ Global } & Basal & 169 & 79.9 & 7.6 & - \\
\hline & Month 3 & 120 & 80.1 & 13.1 & 0.9536 \\
\hline & Month 6 & 83 & 79.0 & 14.9 & 0.2837 \\
\hline \multirow[t]{3}{*}{ Hormone sensitive (HS) } & Basal & 58 & 81.6 & 7.2 & - \\
\hline & Month 3 & 46 & 81.0 & 15.3 & 0.6883 \\
\hline & Month 6 & 36 & 83.1 & 10.9 & 0.6424 \\
\hline \multirow[t]{3}{*}{ Hormone resistant (HR) } & Basal & 111 & 79.1 & 7.7 & - \\
\hline & Month 3 & 74 & 79.6 & 11.6 & 0.8727 \\
\hline & Month 6 & 47 & 76.0 & 16.9 & 0.0923 \\
\hline
\end{tabular}

aln some cases no data were available.

${ }^{b}$ Comparison with Karnofsky basal visit score (Wilcoxon test).

Abbreviation: SD, standard deviation.

bisphosphonates can play a significant role (Gálvez 1998; Cózar Olmo et al 1999; Coleman 2000). First-generation bisphosphonates showed slight analgesic effectiveness in bone pain in patients with bone metastasis resulting from prostate cancer. Saad et al (2002) was the first to demonstrate the usefulness of the zoledronic acid in these patients.

The results of the present study confirm and complement those of Saad et al (2002). We analyzed additional variables such as pain at rest, pain on movement, ECOG scale of functional status, a mixed scale such as Lattinen that evaluates pain, sleep, and functional status, and a QoL questionnaire.

The at-rest VAS, as with the on-movement scores, improved relative to baseline. These differences were statistically different, albeit moderate in absolute terms within the first month of treatment and over the whole 6-month follow-up period. Patients valued this factor highly, since the stability and some improvement in functional status were

Table 9 Adverse reactions recorded

\begin{tabular}{|c|c|c|c|c|c|c|c|c|c|}
\hline \multirow[t]{2}{*}{ Type } & \multicolumn{3}{|c|}{ Total } & \multicolumn{3}{|c|}{ Hormone resistant (HR) } & \multicolumn{3}{|c|}{ Hormone sensitive (HS) } \\
\hline & NR & NP & $\%$ & NR & NP & $\%$ & NR & NP & $\%$ \\
\hline Muscle-skeletal & 7 & 7 & 3.3 & 3 & 3 & 1.4 & 3 & 3 & 1.4 \\
\hline Arthralgia & 3 & 3 & 1.4 & 1 & I & 0.5 & 2 & 2 & 0.9 \\
\hline Myalgia & 2 & 2 & 0.9 & 1 & 1 & 0.5 & 0 & 0 & 0.0 \\
\hline Bone pain & 2 & 2 & 0.9 & 1 & 1 & 0.5 & 1 & 1 & 0.5 \\
\hline Nervous system & 4 & 4 & 1.9 & 1 & 1 & 0.5 & 3 & 3 & 1.4 \\
\hline Dizziness & 3 & 3 & 1.4 & 1 & I & 0.5 & 2 & 2 & 0.9 \\
\hline Neuralgia & 1 & 1 & 0.5 & 0 & 0 & 0.0 & 1 & I & 0.5 \\
\hline Gastrointestinal & 14 & II & 5.2 & 8 & 6 & 2.8 & 4 & 4 & 1.9 \\
\hline Constipation & 4 & 4 & 1.9 & 1 & 1 & 0.5 & 3 & 3 & 1.4 \\
\hline Diarrhea & 1 & 1 & 0.5 & 1 & I & 0.5 & 0 & 0 & 0.0 \\
\hline Vomiting & 5 & 5 & 2.4 & 4 & 4 & 1.9 & 0 & 0 & 0.0 \\
\hline Nausea & 4 & 4 & 1.9 & 2 & 2 & 0.9 & 1 & 1 & 0.5 \\
\hline General body & 20 & 15 & 7.1 & 9 & 6 & 2.8 & 11 & 9 & 4.2 \\
\hline Edema & 3 & 3 & 1.4 & 2 & 2 & 0.9 & 1 & I & 0.5 \\
\hline Asthenia & 2 & 2 & 0.9 & 2 & 2 & 0.9 & 0 & 0 & 0.0 \\
\hline Fever/shivering & 5 & 5 & 2.4 & 3 & 3 & 1.4 & 2 & 2 & 0.9 \\
\hline Generalized illness & 2 & 2 & 0.9 & 1 & I & 0.5 & 1 & $\mathrm{I}$ & 0.5 \\
\hline Generalized pain & 3 & 3 & 1.4 & 1 & 1 & 0.5 & 2 & 2 & 0.9 \\
\hline Influenza-type symptoms & 4 & 4 & 1.9 & 0 & 0 & 0.0 & 4 & 4 & 1.9 \\
\hline
\end{tabular}

Abbreviations: NR, number of adverse reactions; NP, number of patients with adverse reactions (some patients had more than one adverse reaction). 
GLOBAL ( $n=21$ events) HORMO-SENSITIVE $(n=6$ events) HORMO-RESISTANT $(n=15$ events)

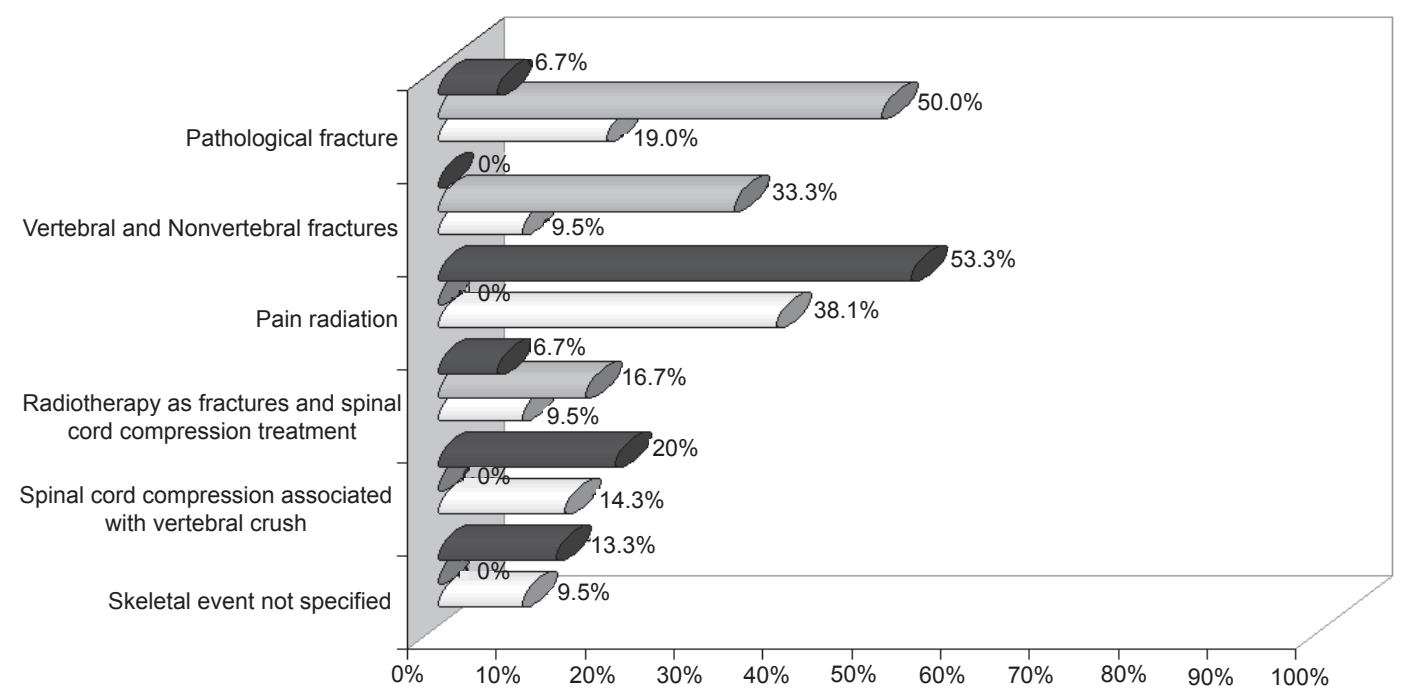

Figure I Skeleton-related events (SRE).

perceived as decisive QoL factors. This same perception can be applied to the attending physicians (oncology, urology, pain units) who consider that pain from bone metastasis is difficult to treat because of the poor response to analgesics. The result is a progressive loss of QoL. As such, stabilization over 6 months with zoledronic acid appears to be a big advance in terms of analgesia and QoL (Cherny and Portenoy 1994; Cózar Olmo et al 1999). These data are confirmed by comparing ECOG and KI status at baseline and end of study at 6 months.

Study patients were hormone-sensitive and hormonerefractory, which is a major difference from the study of Saad et al (2002). Our results showed that zoledronic acid behaves similarly under both conditions.

As has been cited by various authors (Berruti et al 2001; Scher 2003; Papapoulos et al 2000; Saad et al 2002), the use of bisphosphonates in patients with bone metastasis from prostate cancer has two main objectives: to reduce pain and to help avoid bone fractures and adverse skeletal events. Skeletal lesions appear more frequently with the hormonal treatment employed in these patients because of the resultant loss in bone mass which, in addition to the metastasis, makes fractures more likely (Daniell 1997; Townsend et al 1997; Smith et al 2001).

Zoledronic acid needs to be an integral part of a multi-disciplinary treatment plan for bone metastasis resulting from prostate cancer, and should be the fruit of the collaboration between urologists, oncologists, radiologists, and pain management specialists (Berenson 2005).
In addition to this drug, radiotherapy or use of radioactive isotopes (such as strontium) may be indicated as complement to an appropriate analgesic (Gálvez 1998; Cózar Olmo et al 1999; Schmeler and Bastin 1996).

The low frequency of adverse effects (fever, arthralgia, constipation) and good tolerability observed with zoledronic acid in our study appeared not too different from that observed with other bisphosphonates, particularly that of pamidronate, and with less severe side-effects at the pharmacologically efficacious dose of $4 \mathrm{mg}$ iv a month. Similarly, although the parenteral route is less convenient, fewer digestive-tract disruptions occur than when taken orally (Adami and Zamberlan 1996; Saad et al 2002; Zojer et al 1999). Generalized arthralgias occur for around 48 hours and are alleviated, or pre-empted, with paracetamol at a dose of $3 \mathrm{~g} /$ day. Renal function needs to be monitored. Our one patient who suffered renal dysfunction had been recruited despite having an elevated creatinine level. The renal dysfunction became apparent after the first dose of the zoledronic acid.

The need for an iv dose administration of only once every 4 weeks was perceived as comfortable and acceptable by most patients, and was a deciding factor in patients wishing to continue with this treatment.

In conclusion, we have demonstrated analgesic benefit and good tolerability of zoledronic acid at the doses indicated in these patients with bone metastasis. The results confirm and extend previous findings. The reduced dose, ease of management, and periodicity of use promote zoledronic acid 
as a first-line drug in the management of patients suffering from advanced prostate cancer and bone metastasis.

\section{Disclosures}

None of the authors have any conflicts of interest to disclose.

\section{References}

Adami S, Zamberlan N. 1996. Adverse effects of bisphosphonates. A comparative review. Drug Safety, 14:158-70.

Averbuch S. 1993. New bisphosphonates in the treatment of bone metastasis. Cancer, 72(Suppl):3443-52.

Berenson JR. 2005. Recommendations for zoledronic acid treatment of patients with bone metastasis. Oncologist, 10:52-62.

Berruti A, Dogliotti L, Tucci M, et al. 2001. Metabolic bone disease induced by prostate cancer: rationale for the use of bisphosphonates. $J$ Urol, 166:2023-31.

Boissier S, Ferreras M, Peyruchaud O, et al. 2000. Bisphosphonates inhibit breast and prostate carcinoma cell invasion, an early event in the formation of bone metastasis. Cancer Res, 60:2949-54.

Bruera E. Organizing palliative care. Cancer Strategies for the New Millennium [online]. WHO publications. URL: http:/www.who.int/ ncd/cancer/publications/abstracts/abs9810 22.html.

Cherny NI, Portenoy RK. 1994. Cancer pain. Principles of assesment and syndromes. In: Wall PD, Melzack R (eds). Texbook of pain. 3rd edition. Edinburgh: Churchill Livingstone. pp. 787-823.

Clamp A, Danson S, Nguyen H, et al. 2004. Assessment of therapeutic response in patients with metastatic bone disease. Lancet Oncol, 5:607-16.

Clarke NW, McClure J, George NJ. 1991. Morphometric evidence for bone resorption and replacement in prostate cancer. Br J Urol, 68:74-80.

Coleman RE. 1991. Bisphosphonate treatment of bone metastasis and hypercalcemia of malignancy. Oncology, 5:55-60.

Coleman RE. 2000. Optimising treatment of bone metastasis by Aredia ${ }^{\text {тм }}$ and Zometa ${ }^{\mathrm{TM}}$. Breast Cancer, 7:361-9.

Coleman RE. 2001. Metastatic bone disease: clinical features, pathophysiology and treatment strategies. Cancer Treat Rev, 27:165-76.

Cózar Olmo JM, Gálvez Mateos R, Tallada Buñuel M. 1999. Modalidades terapéuticas en el dolor óseo del cáncer de próstata. Actualidad en Urología. Barcelona. Redacción Médica.

Daniell HW. 1997. Osteoporosis after orchiectomy for prostate cancer. J Urol, 157: 439-44.

Dreicer R. 1997. Metastatic prostate cancer: assessment of response to systemic therapy. Semin Urol Oncol, 15:28-32.

Gálvez R. 1998. Tratamiento del dolor óseo metastásico en el cáncer de próstata. Urol Integr Invest, 3:321-9.
Garnero P, Buchs N, Zekri J, et al. 2000. Markers of bone turnover for the management of patients with bone metastasis from prostate cancer. Br J Cancer, 82:858-64.

Levy MH. 1993. Medical management of cancer pain. In: Warfield CA (ed). Principles, practice of pain management. New Cork: McGraw Hill. pp. 235-50.

Major PP, Lipton A, Berenson J, et al. 2000. Oral bisphosphonates: A review of clinical use in patients with bone metastasis. Cancer, 88:6-14.

Oh WK. 2000. Chemotherapy for patients with advanced prostate carcinoma. Cancer, 8:3015-21.

Papapoulos SE, Handy NA, Van der Pluijm G. 2000. Bisphosphonates in the management of prostate carcinoma metastatic to the skeleton. Cancer, 88(Suppl):3047-53.

Payne R. 1993. Pain management in the patient with prostate cancer. Cancer, 71(Suppl):1131-7.

Rhiner M, Kedziera P. 1999. Managing breakthrough cancer pain: a new approach. Home Health Nurse, 17(Suppl 1-12):13-5.

Rogers MJ, Watts DJ, Russell RGG. 1997. Overview of bisphosphonates. Cancer, 80:1652-7.

Saad F, Gleason DM, Murria R, et al. 2002. and Zoledronic Acid Prostate Cancer Study Group. Randomized placebo-controlled trial of zoledronic acid in patients with hormone-refractory metastasic prostate carcinoma. J Natl Cancer Inst, 94:1458-68.

Saad F, Gleason DM, Murria R, et al. 2002. and Zoledronic Acid Prostate Cancer Study Group. Long-term efficacy of zoledronic acid for the prevention of skeletal complications in patients with metastatic hormonerefractory prostate cancer. $J$ Natl Cancer Inst, 96:879-82.

Scher H. 2003. Prostate carcinoma: defining therapeutic objectives and improving overall outcomes. Cancer, 97:758-71.

Schmeler K, Bastin K. 1996. Strontium 89 for syntomatic metastatic prostate cancer to bone: recommendations for hospice patients. Hosp J, 11:1-10.

Small EJ, Seaman JJ, Petrone S, et al. 2003. Combined analysis of two multicenter, randomized, placebo-controlled studies of pamidronate disodium for the palliation of bone pain in men with metastatic prostate cancer. Clin Oncol, 21:4277-84.

Smith MR, McGovern FJ, Zietman AL, et al. 2001. Pamidronate to prevent bone loss during androgen-deprivation therapy for prostate cancer. N Engl J Med, 345:948-55.

Tassone P, Forciniti S, Galea E, et al. 2000. Growth inhibition and synergistic induction of apoptosis by zoledronate and dexamethasone in human myeloma cell lines. Leukemia, 14:841-4.

Townsend MF, Sanders WH, Northway RO, et al. 1997. Bone fractures associated with luteinizing hormone-releasing hormone agonists used in the treatment of prostate carcinoma. Cancer, 79:545-50.

Warrel RP. 1992. Etiology and current management of cancer-related hypercalcemia. Oncology, 6:37-43.

Zojer N, Keck AV, Pecherstorfer M. 1999. Comparative tolerability of drug therapies for hypercalcaemia of malignancy. Drug Saf, 21:389-406. 